\title{
Arcillas fibrosas anómalas en encostramientos y sedimentos superficiales: características y génesis (Esquivias, Cuenca de Madrid)
}

\author{
M. A. BUSTILLO', E. GARCÍA ROMERO² \\ 'Dpto. de Geología. Museo Nacional de Ciencias Naturales. CSIC. 28006 Madrid. \\ ²Dpto. de Cristalografía y Mineralogía. Facultad de Ciencias Geológicas. UCM. 28014 Madrid.
}

\begin{abstract}
Se estudian silcretas, calcretas/calizas palustres y niveles arcillosos de zonas continentales superficiales con objeto de caracterizar la mineralogía de las arcillas fibrosas, su composición química y las condiciones de formación. La caracterización mineralógica, petrológica y geoquímica se realiza con difracción de Rayos X (DRX), microscopía óptica, microscopía electrónica de barrido (MEB) y de transmisión (MET) que llevan asociados espectrómetros de energías dispersivas (EDS).

En los niveles arcillosos puros y en los encostramientos siliceos las arcillas fibrosas son paligorskita y sepiolita, mientras que en las calcretas/calizas palustres normalmente solo aparece paligorskita. Ambas arcillas presentan anomalías en su composición, siendo las sepiolitas más ricas en Al que lo usual y las paligorskitas más magnésicas. En los niveles arcillosos la presencia de vestigios de esmectitas puede indicar que los minerales fibrosos resultan de ellas por disolución y posterior precipitación, mientras que en los encostramientos, la ausencia de esmectitas, puede indicar que se forman directamente a partir de fluidos intersticiales. En las calcretas/calizas palustres parte de la paligorskita es posterior a la calcita y su proceso de formación estaría favorecido por procesos de disolución de la calcita dentro de la calcreta /caliza palustre. Las silcretas están formadas por un proceso de silicificación de las calcretas/calizas palustres. La paligorskita es un relicto de la roca original, mientras que la sepiolita es un mineral neoformado junto con el ópalo durante el proceso de silicificación, o algo después, durante el envejecimiento de un gel sílico-magnesiano.
\end{abstract}

Palabras clave: Sepiolita, paligorskita, silicificación, ambiente superficial, geles siliceos.

\section{Uncommon fibrous-clays in duricrust and near surface sediments: features and genesis (Esquivias, Madrid basin)}

Mineralogy, chemical composition and textures of silcretes, calcretes/palustrine limestones and argillaceous beds are studied to consider the features and the genesis of fibrous-clay minerals. The mineralogy, petrology and geochemical studies were carried out by X-ray diffraction, optical microscopy, and, scanning (SEM) and transmission (TEM) electron microscopy with dispersive analysis (EDS). Sepiolite and palygorskite were found in the argillaceous beds and silcretes while only palygorskite was found in the calcretes/palustrine limestones. The chemical compositions of sepiolite and palygorskite are anomalous. The sepiolite shows $\mathrm{Mg} / \mathrm{Al}$ relations lower than the standard, and the palygorskite higher than standard.

Smectites occurs in the argillaceous beds, and the dissolution of these smectites can generate appropriated cations for the formation of sepiolite and palygorskite. The absence of smectites in the silcretes and calcretes/palustrine limestones could suggest that the fibrous clay minerals were formed by direct precipitation from interstitial water of these rocks. Part of the palygorskite included in the calcretes/palustrine limestones formed latter than calcite, and its formation could have been favored by the dissolution of the calcite.

The silcretes are mainly constituted of opal-CT, palygorskite and sepiolite. They are formed by silicification of the calcrete/ palustrine limestones and the palygorskite is the relic of these host rocks. Opal CT and sepiolite are neoformed minerals which were produced during the silicification, or after, by aging from a magnesium rich-silica gel.

Key words: Sepiolite, palygorskite, silicificación, terrestrial environment, silica gels.

\section{INTRODUCCIÓN}

En este trabajo se hace un estudio mineralógico y geoquímico de una secuencia continental de ambiente palustre donde aparecen niveles de arcillas, calcretas/calizas palustres, y silcretas (ópalos). Los niveles de calcretas/calizas palustres y silcretas presentan como característica común el que son muy ricos en minerales fibrosos de la arcilla $(1,2)$.

La aparición de minerales fibrosos en calcretas y calizas palustres es un hecho frecuente cuando el clima es árido (3). Así mismo, los estudios petrológicos sobre silcretas en las cuencas terciarias del Duero y Tajo, han revelado que en muchas silcretas formadas por ópalo aparecen, en mayor o menor proporción, arcillas magnesianas fibrosas $(4,5,6,7)$.
Una revisión de diferentes trabajos sedimentológicos y mineralógicos sobre los minerales fibrosos de las arcillas revela que, en unas ocasiones, su génesis es por procesos de trasformación a partir de silicatos anteriores (principalmente esmectitas) $(8,9,10,11) \mathrm{y}$, en otras, por procesos de precipitación directa a partir de soluciones $(12,13,14,15)$.

Si consideramos los medios de formación, vemos que en sedimentos continentales estas arcillas se asocian a medios lacustres, donde se forman tanto por precipitación química directa como por transformaciones diagenéticas ( 16, 17, 18 ). Pero, el medio en el que se hallan con más frecuencia es en calcretas, caliches o suelos ricos en carbonatos, estando una 
gran parte de los casos, asociadas a carbonatos y/o yesos (12, $19,20,21)$.

El objetivo de este trabajo es determinar las características de los diferentes minerales fibrosos de la arcilla que aparecen en el perfil geológico considerado, estableciendo su génesis y relacionándolos con la formación de encostramientos (calcretas y silcretas). Se comparan las características mineralógicas y geoquímicas de estos minerales en función de su entorno de constitución (nivel de arcilla, calcreta/caliza palustre o silcreta), intentando dilucidar si estos medios de constitución influyen en dichas características. A su vez, se va a establecer la fuerte interrelación que existe entre ciertos minerales fibrosos de la arcilla con las fases opalinas en las silcretas. Este trabajo ha permitido además conocer la existencia de arcillas magnesianas fibrosas de composición anómala, hasta ahora no descritas en la Cuenca de Madrid (22).

\section{MATERIALES Y MÉTODOS}

Este estudio parte de dos muestreos en lateral realizados en canteras de arcillas del Mioceno, próximas a Esquivias (Toledo)(Fig. 1). Su objetivo era dilucidar transformaciones mineralógicas por lo que las columnas litológicas son de poca extensión y los muestreos muy de detalle para poder precisar los cambios (Fig. 2). Desde el punto de vista geológico las secuencias sedimentarias de esta zona han sido interpretadas como secuencias de ambiente lacustre-palustre $(1,2,23)$, considerándose que los ópalos que vamos a estudiar son silcretas (2), mientras que las calizas son calcretas o bien calizas lacustres-palustres transformadas en ambiente subaereo.

En el perfil geológico considerado existen arcillas fibrosas magnesianas en tres situaciones diferentes (Fig. 2): i) formando estratos o capas sedimentarias horizontales, ii) dispersas, y en concentraciones variables, dentro de calizas que también aparecen en estratos y que son interpretadas como calcretas o calizas palustres, iii) homogéneamente dispersas dentro de nódulos y niveles lenticulares ópalinos que han sido considerados silcretas (Fig. 3.a). Estas silcretas están incluidas en las calizas referidas y su formación es por procesos de silicificación (2).

La caracterización mineralógica se ha llevado a cabo con un difractómetro SIEMENS D-500, con radiación $\mathrm{Cu} \mathrm{K \alpha} \mathrm{y}$ monocromador de grafito. En los casos en que se contaba con muestras arcillosas puras se han preparado agregados orientados secados al aire, solvatados con etileglicol y tratados térmicamente $\left(500{ }^{\circ} \mathrm{C}\right.$ durante 2 horas) sobre fracciones finas $(<2 \mu \mathrm{m})$. Cuando las muestras arcillosas se encontraban silicificadas han sido estudiadas únicamente en muestras de polvo, si bien, dado que se trata de arcillas fibrosas (sepiolita y paligorskita), que no se orientan con la preparación de agregados orientados, no hay diferencias significativas. Las muestras de polvo han sido rodadas entre 2 y $65^{\circ} 2 \theta$ y los agregados orientados entre 2 y $30^{\circ} 2 \theta$.

La cuantificación mineralógica de las muestras de arcillas se ha realizado en los difractogramas de agregado orientado, con los poderes reflectantes de 1 para la sepiolita y 0.75 para la paligorskita (24), sin embargo, las calizas y ópalos se han cuantificado a partir de los diagramas de polvo. En ellas, el procedimiento seguido ha consistido en una valoración de las intensidades relativas de las reflexiones diagnóstico de la sepiolita (reflexión a $12 \AA$ ), paligorskita (reflexión a $10.5 \AA$ ) y del ópalo-CT (reflexión a 4.1 Å). Para realizar dicha valoración,

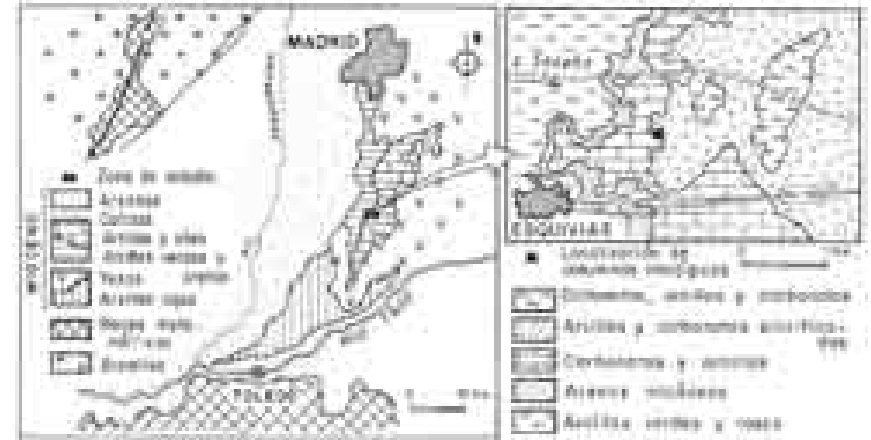

Fig. 1- Mapa de situación general y esquema geológico donde se encuentra la zona estudiada. El esquema esta modificado de Bellanca et al., 1992.

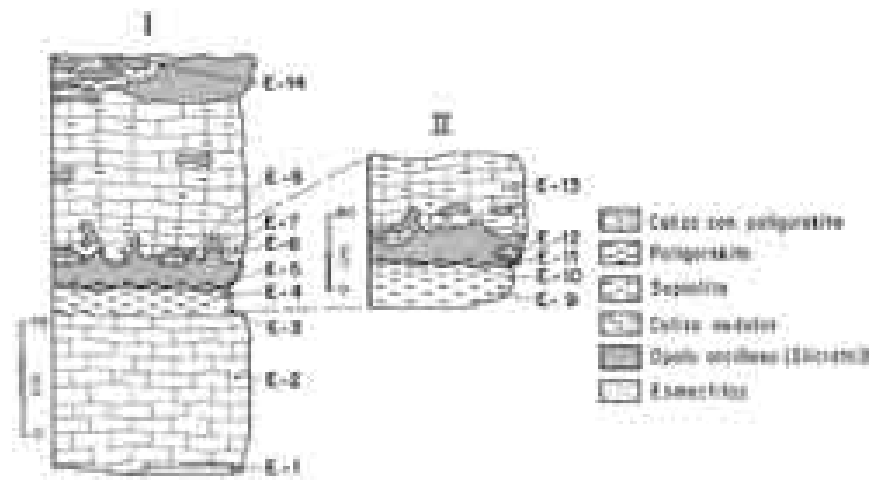

Fig. 2 - Secciones levantadas y situación de las muestras de estudio.

las intensidades fueron corregidas con unos parámetros de referencia calculados a partir de mezclas realizadas con minerales puros de la misma zona. Así, la sepiolita y la paligorskita mezcladas a partes iguales mostraban la misma intensidad, mientras que el ópalo CT la presentaba mayor, por lo que se corrigió dividiendo el valor de su intensidad por 1.37.

Las calizas y ópalos fueron estudiados inicialmente por microscopía óptica de luz polarizada, con objeto de seleccionar los puntos a estudiar con microscopía electrónica. Cuando en las muestras aparecían ópalo y caliza en contacto, se realizaron láminas delgadas en la zona de tránsito entre ambos, ya que en esta zona se puede ver mas claramente la transformación desde la roca carbonática hasta la silcreta resultante.

El estudio de las microtexturas se ha hecho con microscopía electrónica de barrido (MEB) y de transmisión (MET). Las observaciones de MEB se han realizado con un equipo JEOL JSM 6400 operado a $20 \mathrm{kV}$ y equipado con un espectrómetro de energías dispersivas de rayos $\mathrm{X}$ marca Link (EDS). Las observaciones de MEB se llevaron a cabo sobre superficies de fractura fresca de pequeños fragmentos de roca, cubiertos por una película de $\mathrm{Au}$, bajo condiciones de vacío en atmósfera de Ar. Las observaciones de MET se llevaron a cabo sobre partículas dispersas depositadas en rejilla con collodión y recubiertas con oro, con un equipo JEOL 2000 FX, operando a 200 kV. Este microscopio tenía incorporado un espectrómetro de energías dispersivas de rayos-X marca OXFORD ISIS y software para análisis cuantitativos. 


\section{RESULTADOS}

\subsection{Mineralogía}

La composición mineralógica global se expresa en la tabla I. El cuarzo aparece siempre en cantidades inferiores o iguales al $5 \%$, por lo que no se ha expresado en esta tabla. Las muestras de calizas y ópalos pueden tener dos o más análisis diferentes porque son muy heterogéneas. Un ejemplo de ello se ha expresado en la figura 3.b donde se ha marcado la situación de los análisis para E-12 (puntos 1,2,3,4). Según la parte analizada podemos encontrar caliza, paligorskita, $\mathrm{u}$ ópalos de diferente composición (tabla I), y todo ello en una muestra de $8 \mathrm{~cm}$.

Las calizas palustres de la base del perfil están formadas únicamente por calcita, y en ellas no aparece ningún tipo de arcilla fibrosa. Sobre ellas aparecen niveles arcillosos muy puros, formados por sepiolita, paligorskita o mezcla de ambos y con menores cantidades de esmectitas trioctaédricas en su base (tabla I). Las muestras situadas en las calizas superiores (Fig. 2) están formadas por cantidades muy variables de calcita y paligorskita, con proporciones muy pequeñas de cuarzo. Aunque en algunas muestras aparezcan valores altos de calcita (100\%) o de paligorskita ( $85 \%$ ), lo más frecuente son mezclas con valores intermedios (tabla I). Cuando estas calizas con paligorskita, están recogidas muy cerca de las silcretas, presentan también procesos parciales de silicificación, encontrándose en los análisis mineralógicos proporciones pequeñas de ópalo y /o sepiolita.

En las silcretas, el ópalo CT se mezcla en proporciones variables con paligorskita y sepiolita. La calcita es solo ocasional y el cuarzo aparece siempre muy minoritariamente.

Salvo una excepción (tabla I, E-14), al observar comparativamente la mineralogía de las las calcretas / calizas palustres (tabla I, ver E-6, E-7, E-8, E-12 y E- 13) y las silcretas asociadas, se advierte que hay sepiolita en muchas silcretas $y$, sin embargo, no existe en las calcretas / calizas palustres.

La determinación del tipo de ópalo es difícil por la interferencia de las reflexiones de los minerales fibrosos con la de la tridimita ( $4.3 \AA$ ) que forma el interestratificado del ópalo CT. Solo cuando la proporción de ópalo es importante (mas del $50 \%$ ), se puede diferenciar bien la reflexión de la tridimita a $4.30 \AA$ A, confirmándose la presencia de ópalo CT. En el resto de las muestras hay que presuponer que esta es la fase opalina existente, ya que las facies son iguales. La reflexión del ópalo CT correspondiente a la cristobalita varia entre 4.09 y $4.11 \AA$, como suele ser usual en el ópalo CT de las rocas silíceas sedimentarias de ambiente superficial (25). Además, no se puede descartar la presencia, en estas silcretas, de una pequeña cantidad de ópalo A, que siempre puede estar, y aparece enmascarada por el ópalo $\mathrm{CT}$.

\subsection{TEXTURAS Y RELACIONES ENTRE MINERALES}

\subsubsection{Características petrológicas en microscopia óptica}

El estudio en lámina delgada, realizado sobre las silcretas y las calizas que las incluyen, permite encontrar significado a los diferentes minerales hallados en DRX y caracteriza el proceso de silicificación.

Las calizas con paligorskita (calcretas/calizas palustres) definidas en la mitad superior del perfil (Fig. 2) son micritas
TABLA I. COMPOSICIÓN MINERALÓGICA (\%) OBTENIDA POR DiFRACCIÓN DE RX.

\begin{tabular}{|l|l|c|c|c|c|c|}
\hline \multicolumn{1}{|c|}{ LITOLOGía } & MUESTRA & CALCITA & $\begin{array}{c}\text { OPALO } \\
\text { CT }\end{array}$ & SEPIOLITA & $\begin{array}{c}\text { PALIGORS- } \\
\text { KITA }\end{array}$ & ESMECTITA \\
\hline Silcreta & E-14 & 15 & 65 & 5 & 15 & \\
\hline Calcreta */marga & E-14 Rc & 50 & 5 & 15 & 30 & \\
\hline Calcreta*/arcilla & E-13 & 15 & & & 85 & \\
\hline Calcreta*/caliza (1) & E-12 Rc & 85 & & & 15 & \\
Silcreta (2) & E-12 Trn & 25 & 35 & 15 & 25 & \\
Silcreta (3) & E-12 & & 50 & 25 & 25 & \\
Silcreta (4) & E-12.1 & & 30 & & 70 & \\
\hline Nivel de arcilla & E-11 & & & & 100 & \\
\hline Nivel de arcilla & E-10 & & & 100 & & \\
\hline Nivel de arcilla & E-9 & & & 65 & 15 & 20 \\
\hline Calcreta*/caliza & E-8 & 100 & & & & \\
\hline Calcreta*/marga & E-7 & 30 & 10 & & 60 & \\
\hline Silcreta & E-6 & & 50 & 20 & 30 & \\
\hline Calcreta*/marga & E-6 Rc & 30 & 20 & & 50 & \\
\hline Silcreta & E-5.1 & & 40 & 35 & 25 & \\
\hline Silcreta & E-5 & & 25 & & 75 & \\
\hline Nivel de arcilla & E-4 & & & 25 & 70 & 5 \\
\hline Nivel de arcilla & E-3 & & & 45 & 25 & 30 \\
\hline Caliza & E-2 & 100 & & & & \\
\hline Nivel de arcilla & E-1 & & & & 10 & 90 \\
\hline
\end{tabular}

$\mathrm{Rc}=$ roca caja de la muestra con la misma denominación

$\operatorname{Trn}=$ zona de transición entre silcreta y calcreta

* / 8 caliza palustre

y microsparitas, con estructura brechoide o disgregación en ovoides. Localmente se pueden observar bioturbaciones y restos muy esporádicos de diatomeas y espículas silíceas. La paligorskita en lámina delgada no es claramente visible porque la micrita/microsparita la enmascara y no se refleja ni siquiera la estructura fibrosa (Figs. 3.c y 3.d). Las láminas realizadas en la zona de contacto entre la roca caja y la silcreta (Fig. 3.b) revelan que el proceso de silicificación es gradual ya que da lugar a unas aureolas de transición (Figs. 3.b, 3.c y 3.d) y además se conserva perfectamente la estructura nodular y/o brechoide de la roca caja. Las silcretas, al estar formadas por mezclas de ópalo y minerales fibrosos de la arcilla, no son isótropas con nícoles cruzados ( como les correspondería por las fases opalinas) sino que presentan una fuerte birrefringencia estriada (Fig. 3.d), que es el reflejo de la estructura fibrosa de las arcillas. La silicificación reemplaza los carbonatos y resalta la estructura fibrosa de la paligorskita. Por otra parte, al neoformarse sepiolita, como se ha detectado en los análisis mineralógicos, esta nueva arcilla contribuye también a aumentar la microestructura fibrosa.

\subsubsection{CARACTERÍSTICAS MICROMORFOLÓGICAS EN MICROSCOPIA ELECTRÓNICA.}

\subsubsection{Microscopio electrónico de barrido (MEB)}

Cuando se observan las calizas con paligorskita con MEB se advierte su gran heterogeneidad. En ciertos puntos, los cristales de calcita se observan claramente (Fig. 3.e) pero pueden estar enmascarados por la paligorskita. Son cristales euhedrales y subeuhedrales con tamaños entre 1 y $10 \mu \mathrm{m}$. La paligorskita aparece como un entramado de fibras cortas y rectas, a veces con terminaciones curvas adquiriendo aspecto de bastones. Las fibras tienen un tamaño medio en torno a 1 x $0.15 \mu \mathrm{m}, \mathrm{y}$ sólo en raras ocasiones pueden llegar a alcanzar las $2 \mu$ de longitud. La paligorskita frecuentemente tapiza los 

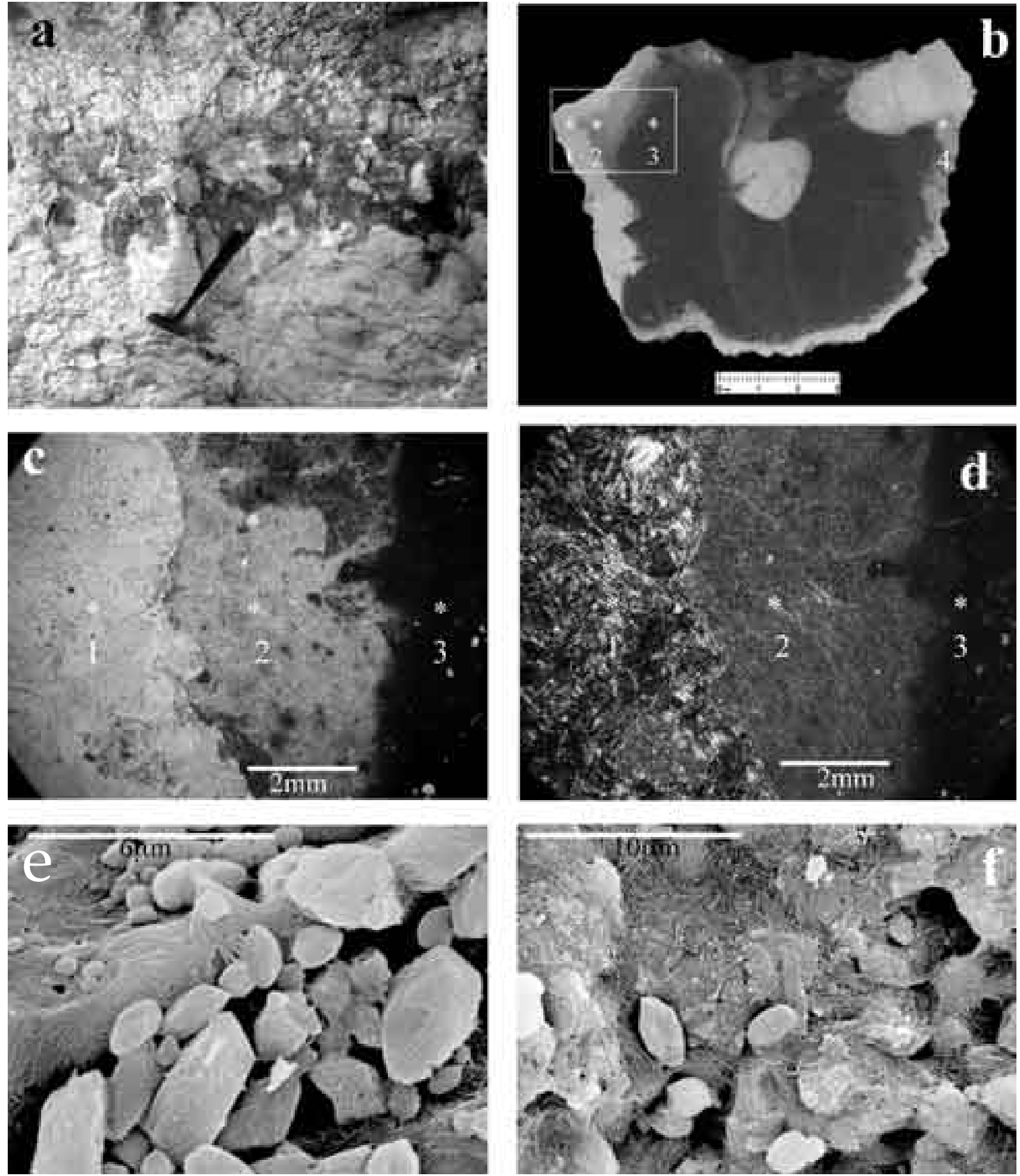

Fig. 3.a) Aspecto de campo del nivel de arcillas fibrosas y, sobre él, la silcreta inferior incluida en calcreta/caliza palustre.

b) Corte de una de las muestras estudiadas, donde se observa un fragmento de silcreta con su roca caja y la zona de transición. En la parte recuadrada fue realizada la lamina delgada a partir de la cual fueron obtenidas las imágenes de las figuras 3.c y 3.d. En la zona 1 esta situada la muestra E-12Rc, en la zona 2 la muestra E-12Tr, en la zona 3 la muestra E-12 y en la zona 4 la muestra E12.1. La composición mineralógica de estas zonas es muy diferente como se refleja en la tabla 1.

c) En microscopia óptica de lamina delgada se diferencia bien el paso gradual de la roca caja (micrita, 3) a la silcreta (ópalo, 1), a través de la zona de transición (2). Nícoles paralelos. Muestra E-12, sección recuadrada de la figura 3.b.

d) Ídem figura 3.c, nícoles cruzados. El ópalo presenta estructura fibrosa por la presencia de las arcillas fibrosas. Estas arcillas se observan también en la zona de transición, pero no en la roca caja.

e) Imagen de las calizas con paligorskita en MEB. Este mineral recubre los cristales de calcita y además se acumula en puntos formando pequeños niveles.

f) Aspecto de una zona de transición. La paligorskita es abundante pero se pueden observar todavía los cristales de calcita. El ópalo no se diferencia claramente pero la composición obtenida por EDAX, reflejaba su presencia. 
cristales de calcita formando un velo alrededor de ellos, otras veces, rellena huecos entre ellos y, en ocasiones, es ya una masa compacta de fibras donde la calcita queda englobada y no se advierte. En las zonas de transición entre la caliza y la silcreta el aspecto es parecido, aunque predomina la estructura fibrosa ( Fig. $3 \mathrm{f}$ ). Todas estas posibilidades pueden observarse dentro de una misma sección $\left(1 \mathrm{~cm}^{2}\right)$ estudiada con MEB.

Los niveles arcillosos aparecen como un gran entramado fibroso donde las fibras crecen apretadamente dando lugar a masas compactas (Fig. 4.a). No se pueden diferenciar entre sí las fibras de sepiolita y paligorskita. Hay que señalar que, en las muestras de sepiolita pura aparecen, en algunas zonas determinadas, numerosas "lepiesferas" de sílice de un tamaño

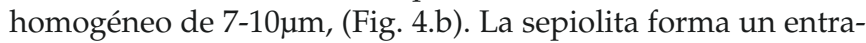
mado de fibras cortas y rectas que constituye bandas enlazando varias "lepiesferas"(Fig. 4.c), o incluso, forma superficies continuas de fibras entrelazadas que recubren totalmente las zonas en las que se acumulan las "lepiesferas".

Cuando se observan con MEB muestras de silcretas se puede apreciar que la silicificación es heterogénea y afecta a la muestra de forma discontinua. Las zonas en las que el proceso de silicificación ha sido completo (los análisis puntuales solo detectan Si y O) son muy compactas y homogéneas, pero el carácter fibroso sigue presente (Fig. 4.d, parte superior). En las zonas en las que el proceso de silicificación es incompleto hay una porosidad mayor ( Fig. 4.d parte inferior) y los análisis químicos puntuales realizados señalan importantes variaciones $\left(\mathrm{SiO}_{2}\right.$ 75.49-89.71\%; $\mathrm{Al}_{2} \mathrm{O}_{3} 1.05-2.86 ; \mathrm{MgO}$ 4.14-6.63; $\mathrm{CaO}$ 5.11-15.02) que evidencian la coexistencia de calcita, arcillas fibrosas y ópalo en proporciones variables. Estas zonas están formadas por un entramado de fibras entre las que, ocasionalmente, se aprecian formas cristalinas semejantes a las de la calcita de las calcretas, completamente recubiertas de fibras (Fig. 4.e). Si comparamos la estructura fibrosa de las zonas silicificadas con los entramados de fibras que aparece en las calcretas/calizas palustres se advierte que, en las partes silicificadas, las fibras son más gruesas y pueden estar constituidas por microesferas (Fig. 4.f).

\subsubsection{Microscopio electrónico de transmisión (MET y EDS)}

Con MET, los cristales de calcita observados en las calizas con paligorskita son euhedrales o subheuedrales (de 1 a $3 \mu \mathrm{m}$ de tamaño). Sobre los cristales de carbonatos se disponen numerosas fibras, de composición paligorskitica, lo que hace que los bordes de los carbonatos, en muchas ocasiones, queden difusos (Fig. 5.a). En otras ocasiones las fibras se distribuyen formando grupos o haces de fibras alrededor de los cristales de calcita. La paligorskita asociada a la calcita en las calcretas / calizas palustres presenta el mismo aspecto que la que forma los niveles arcillosos. Se trata de fibras con longitudes entre 0.5 y $1 \mu \mathrm{m}$, alcanzando solo ocasionalmente $2 \mu \mathrm{m}$. Las fibras, a su vez, generalmente se agrupan formando haces de fibras paralelas que dan lugar a partículas de mayor tamaño (1 a 2 $\mu \mathrm{m}$ ) (Fig. 5.b). Las fibras de sepiolita observadas en los niveles arcillosos constituidos solo por este mineral, muestran mayor tamaño, alcanzando en muchas ocasiones longitudes de $2 \mu \mathrm{m}$, y también se pueden agrupar con el eje c paralelo, dando lugar a partículas mayores (de hasta $3 \mu \mathrm{m}$ de tamaño) (Fig. 5.c), o bien aparecen como fibras sueltas dispuestas en forma desor- denada. En observaciones de detalle se observa que tanto las fibras de sepiolita como las de paligorskita están formadas por agrupaciones de fibras mas pequeñas ( 0.1 a $0.2 \mu \mathrm{m}$ de anchura) dispuestas paralelamente.

La formula cristaloquímica media de la paligorskita es:

$$
\left(\mathrm{Si}_{7.84} \mathrm{Al}_{0.16}\right)\left(\mathrm{Al}_{0.99} \mathrm{Fe}^{3+}{ }_{0.16} \mathrm{Mg}_{3.28}\right) \mathrm{Ca}_{0.01} \mathrm{Na}_{0.08} \mathrm{~K}_{0.05} \mathrm{O}_{20}(\mathrm{OH})_{2}\left(\mathrm{OH}_{2}\right)_{4} \cdot 4 \mathrm{H}_{2} \mathrm{O}
$$

La de la sepiolita es:

$$
\left(\mathrm{Si}_{11.66} \mathrm{Al}_{0.34}\right)\left(\mathrm{Al}_{0.18} \mathrm{Fe}^{3+}{ }_{0.12} \mathrm{Mg}_{5.97}\right) \mathrm{Ca}_{0.16} \mathrm{Na}_{0.09} \mathrm{~K}_{0.01} \mathrm{O}_{30}(\mathrm{OH})_{4}\left(\mathrm{OH}_{2}\right)_{4}
$$

Como puede verse, se trata de una paligorskita con un contenido en $\mathrm{Mg}$ muy alto (22), mientras que, por el contrario, la sepiolita es bastante alumínica, si se compara con los datos de la bibliografía (26).

En las silcretas se pueden observar, además de restos de las calcretas, nuevas partículas de aspecto globular, de 0,5 a $1 \mu \mathrm{m}$, con bordes difusos (Fig. 5.d). Los análisis puntuales obtenidos con EDS indican que químicamente estas partículas están compuestas mayoritariamente por $\mathrm{Si}$, acompañado de cantidades muy inferiores de $\mathrm{Al}$ y Mg (Tabla II). Los análisis puntuales ponen de manifiesto una heterogeneidad en la composición química dentro de la partícula, siendo más alta la proporción de Si en el centro (95.25 a 97.73 \%), aumentando el $\mathrm{Al}$ y el $\mathrm{Mg}$ en la periferia.

\section{DISCUSIÓN DE RESULTADOS}

El perfil geológico de Esquivias permite estudiar minerales fibrosos de la arcilla en diferentes marcos sedimentarios, y establecer relaciones temporales y genéticas entre el conjunto de minerales que aparecen. Los minerales fibrosos unas veces constituyen niveles arcillosos independientes, y otras, forman rocas mixtas con calcita y ópalo CT, y estos escenarios diferentes tienen que ser discutidos independientemente.

En los muestreos del nivel arcilloso superior ( Fig. 2, tabla I) se observa que sepiolita y paligorskita pueden aparecer independientemente o coexistir muy interpenetradas (Fig. 4.a). Estos minerales fibrosos también pueden estar acompañados de proporciones variables de esmectitas de carácter trioctaédrico que desaparecen o son casi imperceptibles (5\%) en el techo del nivel. Según datos sobre la síntesis de arcillas fibrosas, la presencia paligorskita y/o sepiolita en ambientes sedimentarios indicaría un medio hipersalino con alta actividad de $\mathrm{Si}$ y $\mathrm{Mg}$ y elevado $\mathrm{pH}(8-10)(27,28,29)$. La disponibilidad de $\mathrm{Al}$ es la que controla la aparición de sepiolita o paligorskita $(30,13)$. La sepiolita se forma en condiciones de mayor alcalinidad que la paligorskita, donde los niveles de $\mathrm{Mg}$ y Si son altos, pero los de Al son menores (31). En los episodios en los que la disponibilidad de $\mathrm{Al}$ desciende, probablemente tras la formación de paligorskita, tiene lugar la precipitación de sepiolita, incluso pueden coexistir ambas fases y pueden originarse ambos minerales asociados. En el estudio mediante EDS se ha observado que la composición química de las arcillas es anómala. Las paligorskitas son excesivamente ricas en $\mathrm{Mg}$ frente al $\mathrm{Al}$ en la capa octaédrica, mientras que las sepiolitas, sin embargo, son ricas en $\mathrm{Al}$, si se comparan con los datos bibliográficos (26). Curiosamente, ambas fases pueden llegar a tener la misma composición química en EDS. Esto puede ser reflejo de que existe un campo de estabilidad donde coexisten ambas fases. 

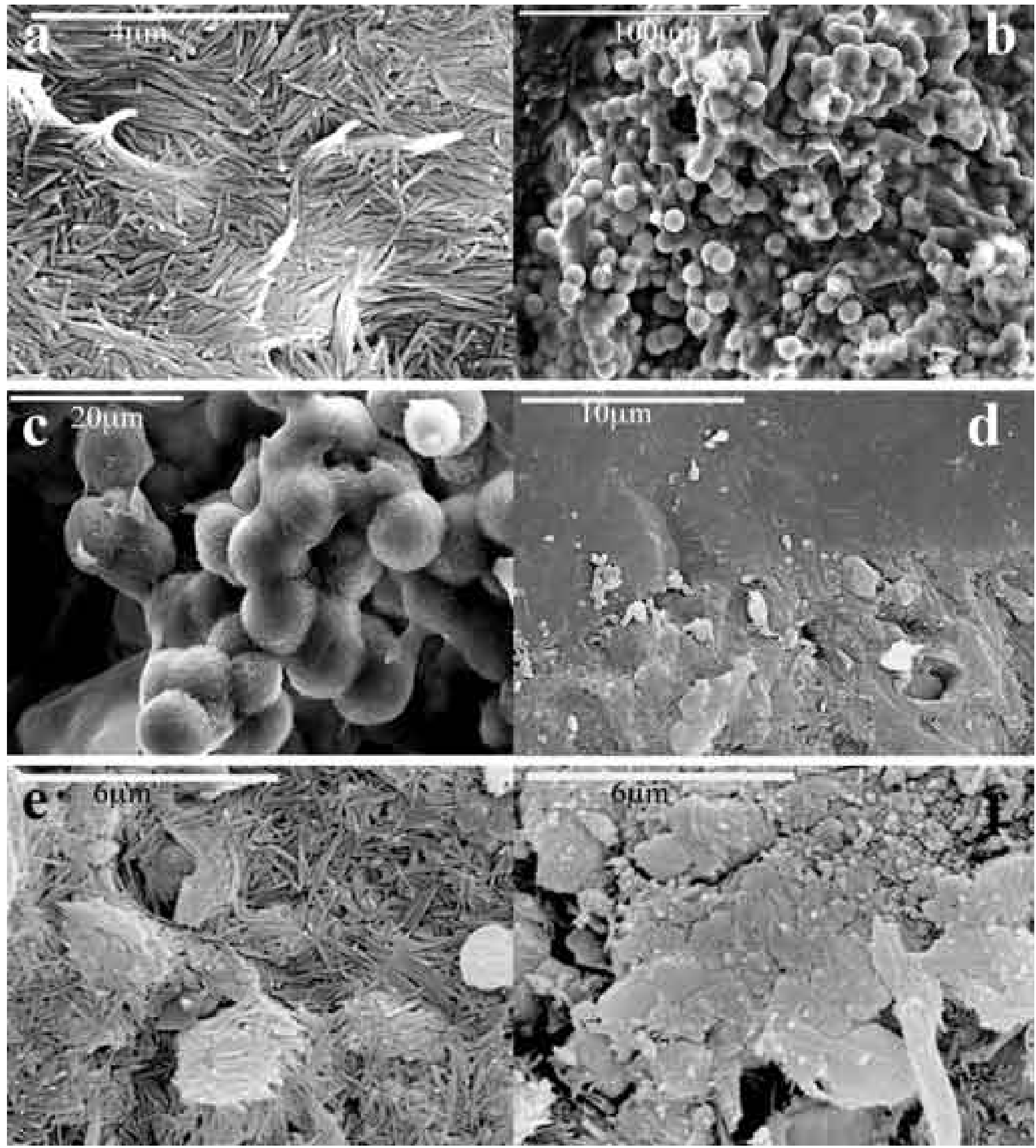

Fig. 4.a) Nivel arcilloso. Masas compactas de paligorskita y sepiolita, en las que no se puede diferenciar ambos minerales. MEB b) Entramados de "lepiesferas" de sílice que aparecen localmente en el nivel de sepiolita. MEB.

c) Las "lepiesferas" estan enlazadas y recubiertas por sepiolita. MEB

d) La silcreta manifiesta variaciones de porosidad, siendo las partes compactas las que corresponden a las zonas mas intensamente silicificadas y que dejan ver peor los minerales. MEB

e) En las partes mas porosas de la silcreta se observa la estructura fibrosa correspondiente a los minerales de la arcilla y también se refleja débilmente algún cristal de calcita. MEB

f) Las fibras que se manifiestan dentro del ópalo son mas gruesas que las normales de una sepiolita y parecen estar formadas por microesferas. MEB 


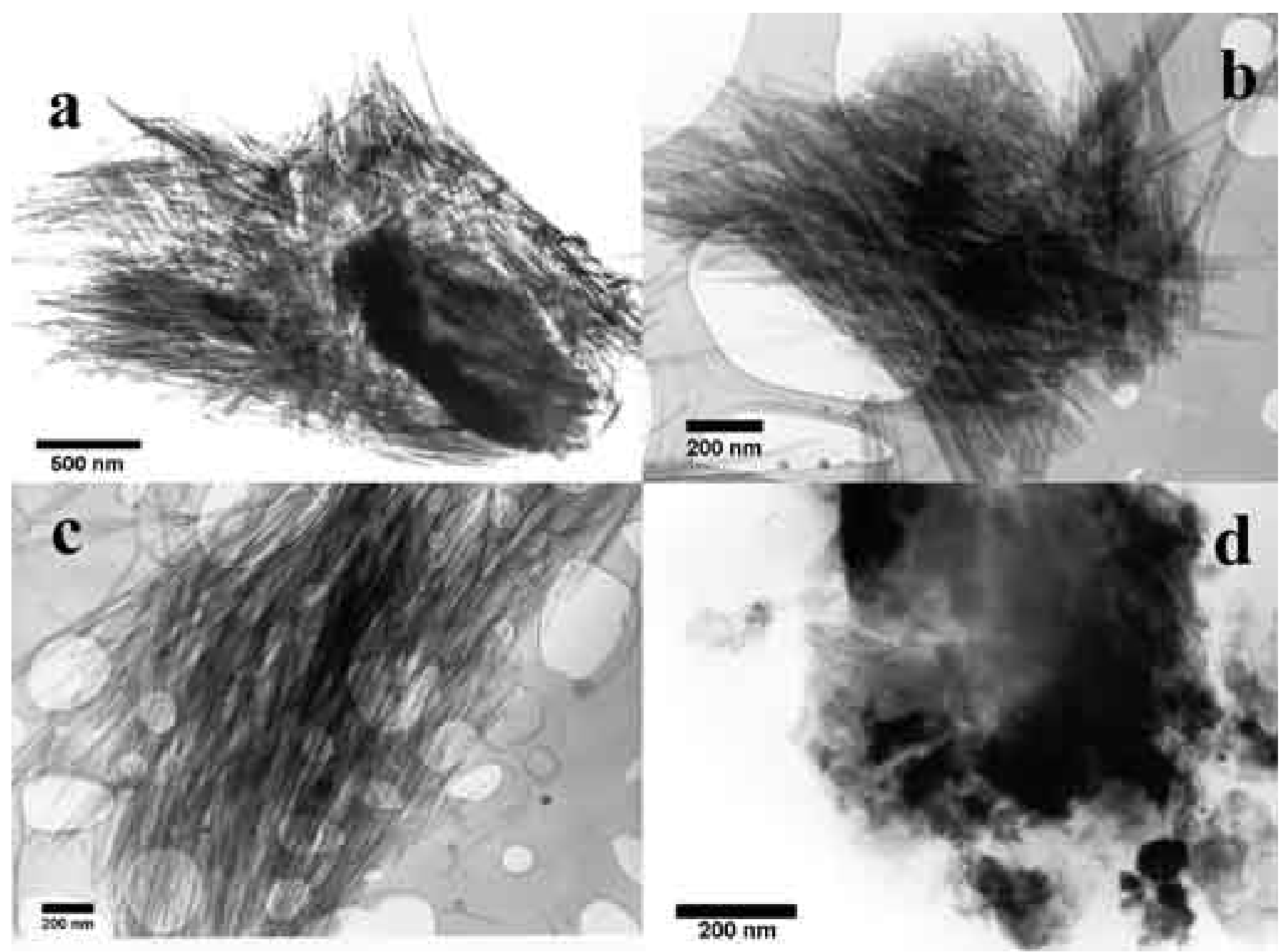

Fig. 5.a) Partícula de calcita completamente recubierta de fibras de paligorskita. MET. b) Partícula formada por haces de fibras de paligorskita. MET. c) Partícula formada por haces de fibras de sepiolita. MET. d) Partícula silícea de aspecto globular. MET

La desaparición de las esmectitas trioctaedricas hacia techo del nivel arcilloso podría indicar una transformación de las esmectitas en minerales fibrosos, tal y como se expone en un estudio anterior (32) sobre esta zona. En dicho estudio se propone que, de acuerdo con el carácter di o trioctaedrico de la esmectita, se propicia la formación de paligorskita o sepiolita respectivamente. En la serie estudiada, esta claro que hay influencia de las esmectitas, porque estas desaparecen al aumentar las arcillas fibrosas, pero las esmectitas que existen son solo trioctaédricas, y no se podría explicar según el razonamiento anterior la formación de paligorskita. Probablemente existe una disolución de esmectitas que aporta, al menos en parte, los cationes necesarios para que tenga lugar la posterior precipitación de paligorskita y/o sepiolita.

Cuando el nivel esta formado únicamente por sepiolita aparecen lepiesferas de sílice, a veces limpias, pero en general, recubiertas por fibras de sepiolita (Figs. 4.b, 4.c). Estas asociaciones de lepiesferas con sepiolita han sido descritas en otros depósitos de sepiolita de Parla (33) y Mara (34), y se producen tardíamente en grietas, poros y otras discontinuidades de la roca. No son otras formas de sepiolita, como se han interpretado anteriormente (35).

Para las calizas con paligorskita de la columna hay que plantear una interpretación algo diferente. La brechificación y nodulización de la roca indica un ambiente superficial no freático, por lo que podría tratarse de zonas de playa, siendo difícil determinar si son calizas palustres o calcretas con paligorskita. Los diferentes análisis mineralógicos y las observaciones con $\mathrm{MEB}$, revelan que las proporciones de calcita y paligorskita varían mucho dentro de estas facies. En una misma muestra se puede observar como la paligorskita pasa de ser un recubrimiento de los cristales de calcita a formar masas compactas donde la calcita prácticamente no existe. Esta variabilidad es típica del ambiente palustre-edáfico. La perfecta envoltura que forma la paligorskita alrededor de los cristales de calcita (Figs. 3.e) indica que, al menos en parte, ella es posterior a la calcita. La aparición de paligorskita en calcretas es tan frecuente que a veces se utiliza como criterio de diagnostico de calcretas (36), pero también se forma en ambientes de playa. En ambos casos, la paligorskita se puede originar por transformación de otros minerales silicatados (esmectitas dioctahedricas frecuentemente), o por autigénesis directa, a partir de las soluciones del suelo, en condiciones evaporíticas. En este estudio, al no aparecer relictos de esmectitas podría pensarse que la paligorskita se ha formado directamente. Por otra parte, no puede ser únicamente un proceso de cementación (37) porque hay zonas 
donde la calcita es escasa. Mas bien parece que es posterior a los carbonatos y que se tiene que formar durante su disolución. El equilibrio disolución-precipitación de la calcita mantiene estable el $\mathrm{pH}$ en torno a 8 , lo que favorece la formación de la paligorskita $(8,38,13)$. Con las concentraciones de sílice que se encuentran normalmente en las soluciones del suelo, la paligorskita podría ser estable a $\mathrm{pH}$ 7.7, si la concentración de $\mathrm{Mg}$ es alta, siendo necesario $\mathrm{pH}$ mas altos (hasta 9) cuando las concentraciones de $\mathrm{Mg}$ son bajas (12). Las paligorskitas estudiadas tienen una composición anómalamente rica en $\mathrm{Mg}$, que podría ser indicativa de una gran riqueza en $\mathrm{Mg}$ de las soluciones intersticiales, por lo que estas paligorskitas podrían formarse a partir de pH 7,7. En general, en el Mioceno de la cuenca de Madrid la disponibilidad de $\mathrm{Mg}$ es anormalmente elevada, como lo demuestran los numerosos tipos y abundancia de arcillas magnesianas que aparecen, y las importantes explotaciones de sepiolita.

En calcretas, la paligorskita se puede formar en cualquier momento de su génesis, es consecuencia del mismo proceso de calcretización, y pueden existir reemplazamientos entre la calcita y la paligorskita (36). Todas estas características pueden ser asumidas también para la formación de paligorskita en calizas palustres.

Las silcretas se han formado por silicificación de las facies de calizas con paligorskita, ya que existen aureolas de transición en el límite de las dos litologías (Figs. 3.b, 3.c, 3.d) y además, la silcreta conserva la estructura de la caliza con paligorskita. La paligorskita dentro de la silcreta es solo un relicto de la roca caja. Durante la silicificación la calcita es reemplazada por ópalo, permaneciendo la paligorskita o parte de ella.

El análisis mineralógico comparado entre las muestras de las silcretas y las de las calizas con paligorskita (tabla I) pone de manifiesto que el caso de la sepiolita es diferente porque es un mineral que aparece preferentemente en la silcreta. En trabajos experimentales (39) se ha encontrado que en un medio rico en sílice, y ambiente alcalino, se pueden formar dominios ricos en $\mathrm{Mg}$, produciéndose un mineral similar a la sepiolita, que se presenta como inclusiones dentro de un ópalo pobre en $\mathrm{Mg}$. Se podría pensar entonces, que la sepiolita se neoforma durante el mismo proceso de silicificación de la caliza. Otra posibilidad seria que una vez formado un gel inicial de composición silico- magnesiana en la silicificación, este gel se transformara a ópalo CT y sepiolita durante el envejecimiento posterior. Al no poder incluir el ópalo CT la totalidad del Mg del gel inicial, se nuclearía sepiolita en puntos determinados. Las partículas halladas en TEM, donde el centro es más rico en sílice que la periferia (tabla II), podrían ser partículas en tránsito de envejecimiento.

Existen varias fuentes de sílice probables para estas silcretas. El hecho de que aparezcan diatomeas y espículas silíceas dispersas en algunos tramos indica una posible fuente biogénica. Las espículas silíceas son poco frecuentes en ambiente continental, pero pueden aparecer incluso en suelos encharcados y pantanos (40). Lateralmente hay además depósitos de diatomitas (23), y es conocido que como consecuencia de la expansión y retracción de lagos de poca profundidad, las diatomitas pueden quedar expuestas, sufriendo procesos pedogénicos o de erosión, generándose durante su disolución aguas ricas en sílice. Una fuente inorgánica podría también contribuir a la silicificación porque estas silcretas están en relación con calcretas que generan sílice cuando su constitución es sobre materiales arcillosos o siliciclásticos.
TABlA II. ANÁLISIS DE PARTíCULAS DE ÓPALO MEDIANTE EDS EN TEM.

\begin{tabular}{|c|c|c|c|c|c|c|c|c|}
\hline & $\mathrm{SiO}_{2}$ & $\mathrm{Al}_{2} \mathrm{O}_{3}$ & $\mathrm{MgO}$ & $\mathrm{CaO}$ & $\begin{array}{c}\mathrm{Mg} / \\
\mathrm{Al}\end{array}$ & $\mathrm{Si} / \mathrm{Mg}$ & $\mathrm{Si} / \mathrm{Al}$ & $\mathrm{Al} / \mathrm{Mg}$ \\
\hline \multirow{4}{*}{$\begin{array}{c}\text { Interior de la } \\
\text { partícula }\end{array}$} & 96,91 & 0,82 & 2,09 & & 2,55 & 46,37 & 118,18 & 0,39 \\
\hline & 92,34 & 1,53 & 4,11 & 1,99 & 2,69 & 22,47 & 60,35 & 0,37 \\
\hline & 95,25 & 2,05 & 3,03 & & 1,48 & 31,44 & 46,46 & 0,68 \\
\hline & 97,73 & 0,49 & 1,5 & 0,27 & 3,06 & 65,15 & 199,45 & 0,33 \\
\hline \multirow{6}{*}{$\begin{array}{c}\text { Periferia de la } \\
\text { partícula }\end{array}$} & 95,54 & 0 & 4,96 & & & 19,26 & & 0,00 \\
\hline & 89,11 & 3,42 & 6,89 & 0,58 & 2,01 & 12,93 & 26,06 & 0,50 \\
\hline & 88,26 & 4,53 & 6,42 & & 1,42 & 13,75 & 19,48 & 0,71 \\
\hline & 86,28 & 2,94 & 10,78 & & 3,67 & 8,00 & 29,35 & 0,27 \\
\hline & 72,32 & 10,13 & 17,48 & & 1,73 & 4,14 & 7,14 & 0,58 \\
\hline & 72,54 & 8,25 & 18,59 & & 2,25 & 3,90 & 8,79 & 0,44 \\
\hline
\end{tabular}

\section{CONCLUSIONES}

1) En los niveles arcillosos aparecen paligorskita, sepiolita o ambos, acompañados en su base por esmectitas trioctaedricas. La presencia de esmectitas puede indicar que son precursoras de los minerales fibrosos, incluso porque su disolución aportaría al sistema, parte de los cationes necesarios para la formación de sepiolita, paligorskita o ambas. Las arcillas fibrosas encontradas son atípicas desde el punto de vista de su composición, y ambas fases se acercan químicamente, es decir, las sepiolitas presentan una composición más rica en $\mathrm{Al}$, que lo usual y las paligorskitas son más magnésicas. Los trabajos experimentales sobre síntesis de minerales han determinado los campos de estabilidad de la sepiolita y paligorskita en función del pH, disponibilidad de sílice, magnesio y aluminio, pero estas composiciones anómalas podrían señalar nuevas condiciones de coexistencia.

A pesar de su anómala composición química, dichos niveles arcillosos, podrían tener aplicaciones industriales dentro del campo de los materiales ad/absorbentes, si bien, su escasa potencia, así como la alternancia con niveles de calcretas y silcretas dificulta su extracción, por ello, sería preciso realizar estudios de viabilidad económica.

2) Las calcretas o calizas palustres presentan casi exclusivamente paligorskita. Al no existir ningún vestigio de esmectitas es probable que esta paligorskita se forme directamente a partir de soluciones intersticiales. Aunque podría originarse durante la génesis de las calcretas/calizas palustres, las relaciones texturales indican que, en parte, la paligorskita es posterior y su proceso de formación estaría favorecido por procesos de disolución de la calcita dentro de la calcreta o caliza palustre.

3) Las silcretas son opalinas y se forman por silicificación de las calizas con paligorskita. En ellas coexisten paligorskita y sepiolita. La paligorskita es un relicto de la roca original, mientras que la sepiolita es un mineral neoformado junto con el ópalo. Esta neoformación tendría lugar durante el proceso de silicificación o quizás un poco mas tarde durante el envejecimiento de las primeras fases silíceas formadas. 


\section{AGRADECIMIENTOS}

Este trabajo ha sido financiado por los proyectos PB-980668-CO2-01 y BTE2002-04017-CO2-01 del Ministerio de Ciencia y Tecnología.

\section{BIBLIOGRAFÍA}

1. S. Leguey, M. Pozo, and J. A. Medina. «Polygenesis of Sepiolite and Palygorskite in a Fluvio-Lacustrine Environment in the Neogene Basin of Madrid». Miner. Petrogr. Acta, 29-A, 287-301 (1985).

2. M. A. Bustillo y M. Bustillo . «Características diferenciales e interpretación genética de los ópalos constituidos en sedimentos biosiliceos y ópalos inorgánicos (Esquivias, Cuenca de Madrid)». Boletín Geológico y Minero XCIX-IV, 615-627 (1988).

3. A. Alonso-Zarza. «Paleoenvironmental significance of palustrine carbonates and calcretes in the geological record». Earth Sciences Reviews, 60, 261-298 (2003).

4. M. A. Bustillo Revuelta. «Estudio petrológico de las rocas silíceas miocenas de la Cuenca del Tajo». Estudios Geológicos, 32, 451-497 (1976).

5. M. Rodas, F. J. Luque, R. Mas, and M. G. Garzón. «Calcretes, palycretes and silcretes in the paleogene detrital sediments of the Duero and Tajo bains, central Spain». Clay Minerals, 29, 273-285 (1994).

6. I. Armenteros, M. A. Bustillo, and J. Blanco. «Pedogenic and groundwater processes in a closed Miocene basin (northern Spain) ». Sedimentary Geology, 99,17-36 (1995).

7. M.A. Bustillo and M. Bustillo. Miocene silcretes in argillaceous playa deposits, Madrid Basin, Spain: petrological and geochemical features. Sedimentology, 47, 1023-1039 (2000).

8. D. M. Yaalon, and M. Wieder. «Pedogenetic palygorskite in some arid brown (calciothid) soils of Israel». Clay Minerals, 11, 73-79 (1976).

9. M. Suárez, M. Robert, F. Elsass, and J. M. Martín Pozas. «Evidence of a precursor in the neoformation of palygorskite-New data by analytical electron microscopy». Clay Minerals, 29, 255-264 (1994).

10. J. Torres-Ruiz, A. López-Galindo, J. M. González-López, and A. Delgado. «Geochemistry of Spanish sepiolite-palygorskite deposits: Genetic considerations based on trace elements and isotopes». Geochemical Geology, $112,221-245$ (1994).

11. A. Lopez-Galindo, A. Ben Aboud, P. Fenoll Hach-Ali, and J. Casas Ruiz. «Mineralogical and geochemical characterization of palygorskite from Gabasa (NE Spain). Evidence of a detrital precursor». Clay Minerals, 31, 33 44 (1996).

12. A. Singer, and K. Norrish. «Pedogenetic Palygorskite. Occurrences in Australia». American Mineralogist, 59, 508-517 (1974).

13. N. L. Watts. «Paleopedogenic palygorskite from the basal Permo-Triassic of northwest Scotland». American Mineralogist, 61, 299-302 (1976).

14. J. Estéoule-Choux. «Palygorskite in the Tertiary deposits of the Armorican Massif». 75-85. n: Palygroskite-Sepiolite. Occurrences, Genesis and Uses. Developments in Sedimentology, 37. Elsevier. (1984).

15. W. C. Isphording. «The clays of Yucatan, Mexico: A contrast in genesis. 59-73. En: Palygorskite-Sepiolite» Occurrences, Genesis and Uses. Developments in Sedimentology, 37. Elsevier, (1984)

16. C. E. Weaver. «Origin and geologic implications of the palygorskite of S. E. United States». 39-58. En: Palygorskite-Sepiolite. Occurrences, Genesis and Uses. Developments in Sedimentology, 37. Elsevier. (1984).

17. E. Galán, and A. Castillo. «Sepiolite-palygorskite in Spanish tertiary basins: Genetical patterns in continental environments». 87-124. En: PalygorskiteSepiolite. Occurrences, Genesis and Uses. Developments in Sedimentology, 37. Elsevier. (1984)

18. A. Chahi, B. Fritz, J. Duplay, F. Weber, and J. Lucas «Textural transition and genetic relationship between precursor stevensite and sepiolite in lacustrine sediments (Jbel Rhassoul, Morocco)». Clays and Clay Minerals, 45 [3], 378389 (1997).
19. N.L. Watts. "Quaternary pedogenic calcretes from the Kalahari (southern Africa): mineralogy, genesis and diagenesis». Sedimentology, 27, 661-686 (1980).

20. A. Singer A. «Pedogenic palygorskite in the arid environment». 169-175. En: Palygorskite-Sepiolite. Occurrences, Genesis and Uses. Developments in Sedimentology, 37. Elsevier. (1984).

21. E. P. Verrecchia, and M. N. Le Coustumer. «Occurrence and genesis of palygorskite and associated clay minerals in Pleistocene calcrete complex, Sde Boqer, Negev desert, Israel». Clay Minerals, 31, 183-202 (1996).

22. E. García-Romero, M. Suárez Barrios, y M. A. Bustillo. «Paligorskita magnésica en Esquivias (Cuenca del Tajo). Datos preliminares». 77-80. En: La investigación de arcillas en geología, agricultura, medio ambiente y ciencia de materiales. Sociedad Española de arcillas. (2002).

23. A. Bellanca, J. P. Calvo, P. Censi, R. Neri, and M. Pozo. «Recognition of lake-level changes in Miocene lacustrine units, Madrid Basin, Spain Evidence from facies analysis, isotope geochemistry and clay mineralogy». Sedimentary Geology, 76, 135-153 (1992).

24. E. Barahona. «Arcillas de ladrillería de la provincia de Granada: evaluación de algunos ensayos de materias primas». PhD. Tesis. Univ. de Granada. España. (1974).

25. M. A. Bustillo, R. García, y M. V. G. García Pérez. «Caracterización y respuesta al tratamiento térmico de rocas silíceas opalinas». Bol. Soc. Esp. Cerám. Vidrio, 39, 741-746 (2000).

26. A. C. D. Newman, and G. Brown. «The chemical constitutions of clays». 75-85 In: Chemistry of Clays and Clay Minerals. Mineralogical Society. Monograph $\mathrm{N}^{\circ}$ 6. Longman Scientific \& Tecnical, (1987).

27. B. Siffert and R. Wey. «Synthèse d'une sépiolite à température ordinaire ». C. R. Acad. Scei., Paris, 245, 1460-1463 (1962).

28. R. Wollast, F.T. Mackenzie, and O. Bricker. «Experimental precipitation and genesis of sepiolite at earth-surface conditions». The American Mineralogist, 53, 1645-1662 (1968)

29. A. La Iglesia «Precipitación por disolución homogénea de silicatos de aluminio y magnesio a temperatura ambiente. Síntesis de la paligorskita». Estudios Geológicos, 33, 535-544 (1977).

30. R.L. Hay, and B. Wiggins. «Pellets, ooids, sepiolite and silica in three calcretes of the southwestern United States». Sedimentology , 27, 559-576 (1980).

31. C. E. Weaver and K. C. Beck. «Miocene of the S. E. United States: A model for chemical sedimentation in a peri-marine environment ». Sedimentary Geology, 17, 1-23 (1977).

32. J. L. Martín de Vidales, M. Pozo, J. A. Medina, y S. Leguey. «Formación de sepiolita-paligorskita en litofacies lutítico-carbonáticas en el sector de BoroxEsquivias (Cuenca de Madrid)». Estudios Geológicos, 44, 7-18 (1988).

33. J. A. Medina, J. Santaren, J. A. Martín Rubí, J. Casas, J. Cuevas, A. Alvarez, and S. Leguey. «Characterization of sepiolite from Cerro Cantueña deposits (Parla, Madrid): microstructure and rheological properties». 693-696. In: Current research in Geology applied to ore deposits. Eds: Fenoll Hach-ali, P., Torres-Ruiz, J y Gervilla, F. (1993).

34. M. J. Mayayo, J. Torres- Ruiz, J. M. González-López, Al López-Galindo y B. Bauluz. «Mineralogical and chemical characterization of the sepiolite/Mg smectite deposit at Mara. (Calatayud Basin, Spain)». Eur. J. Mineral, 10, 363383 (1998).

35. A. U. Gehring, P. Keller, B. Frey and J. Lister. «The occurrence of spherical morphology as evidence for changing conditions during the genesis of sepiolite deposits». Clay Minerals, 30, 83-86 (1995).

36. H. Paquet, and N. Clauer. Soils and sediments. Mineralogy and geochemistry. Springer-Verlag. Berlín. 369 pgs. (1997).

37. M. Pozo, J. A. Medina y S. Leguey. «Mineralogénesis de paligorskita en la zona central de la Cuenca de Madrid». Bol. Soc. Esp. Mineralogía, 8, 271-283 (1985).

38. R. K. Stoessell, and R. L. Hay. «The Geochemical Origin of Sepiolite and Kerolite at Amboseli, Kenya». Contrib. Mineral. Petrol, 65, 225-267 (1978).

39. G. O. Nechiporenko, G. P. Bondarenko, and Ye A. Gorshkova. «Interaction of $\mathrm{Mg}$ with dissolved and precipitated silica in sea water». Geochemistry International, 5, 75-83 (1993).

40. J. Clark. «The occurrence and significance of biogenic opal in the regolith». Earth-Science Reviews, 60, 175-194 (2003). 